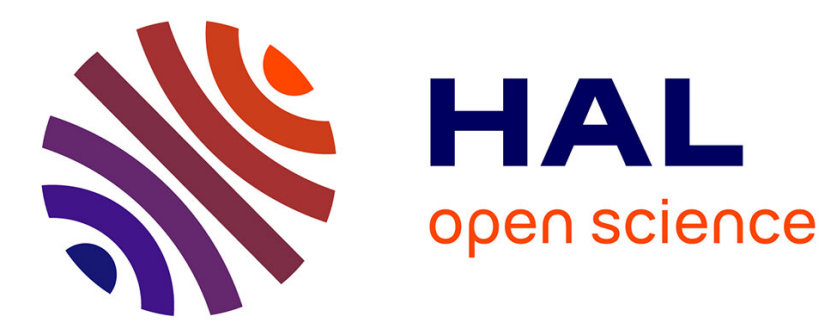

\title{
Influence of Relative Humidity on the Thermomechanical Behavior of PA6.6
}

Adil Benaarbia, André Chrysochoos, Gilles Robert

\section{To cite this version:}

Adil Benaarbia, André Chrysochoos, Gilles Robert. Influence of Relative Humidity on the Thermomechanical Behavior of PA6.6. 2014 Annual Conference on Experimental and Applied Mechanics, Aug 2014, Greenville, United States. pp.167-176. hal-01060991

\section{HAL Id: hal-01060991 \\ https://hal.science/hal-01060991}

Submitted on 15 Sep 2014

HAL is a multi-disciplinary open access archive for the deposit and dissemination of scientific research documents, whether they are published or not. The documents may come from teaching and research institutions in France or abroad, or from public or private research centers.
L'archive ouverte pluridisciplinaire HAL, est destinée au dépôt et à la diffusion de documents scientifiques de niveau recherche, publiés ou non, émanant des établissements d'enseignement et de recherche français ou étrangers, des laboratoires publics ou privés. 


\title{
Influence of Relative Humidity on the Thermomechanical Behavior of PA6.6
}

\author{
Adil Benaarbia, André Chrysochoos, and Gilles Robert
}

\begin{abstract}
An experimental protocol was developed to achieve complete energy balances associated with low cycle fatigue (LCF) of a polyamide 6.6 matrix (PA6.6). The protocol involves quantitative infrared techniques (IRT), and digital speckle image correlation (DIC). IRT data were used with a local heat diffusion equation to estimate strain-induced heat sources, namely dissipation and coupling sources, while DIC enabled strain and stress assessments. Both techniques were then successfully combined to quantify deformation, dissipated and stored energies and then to estimate the Taylor-Quinney ratio that is widely used in plasticity.

In this paper, the effects of loading frequency and relative humidity were investigated. It was shown that an increase of relative humidity resulted in a decrease in the mean stored energy rate per cycle, while the stored energy ratio was much smaller at low than at high loading frequency. In addition, it was found that this ratio could be negative at the last fatigue stage, just before macroscopic crack inception. These energy properties will act safeguards for the future development of a thermomechanical model of PA6.6 matrix behavior.
\end{abstract}

Keywords Infrared thermography $\bullet$ PA6.6 matrix $\bullet$ Relative humidity $\bullet$ Stored energy rate per cycle $\bullet$ Digital image correlation

\subsection{Introduction}

Semi-crystalline polymers represent an important class of materials which are now widespread in various industrial areas. These engineering materials are of particular interest because of their remarkable advantages, notably regarding their low density, high deformability, toughness and long lifespan. Considering the recent growing interest in these engineering materials for automotive structures, it is essential to enhance the overall knowledge of their thermomechanical constitutive behavior during repetitive cyclic loadings. The thermomechanical behavior of these thermo-hygro-sensitive materials can be completely characterized and conceivable when all factors and mechanisms affecting their lifespan are identified. Several deformation mechanisms involved during the fatigue process in such materials have been thoroughly studied in several experimental investigations. However, some physical concepts tightly related to these deformation mechanisms are yet to be completely understood and require full clarification. One promising approach for assessing these concepts is based on energy considerations. A combined description of mechanical and energy phenomena occurring during the deformation process may shed greater light on the behavior of these polymeric materials.

\footnotetext{
A. Benaarbia (凶)

LMGC, CNRS, Université Montpellier 2, Place Eugène Bataillon, 34095 Montpellier, France
}

Solvay Engineering Plastics, Technyl Innovation Center-Simulation et Validation des Applications, Avenue Ramboz - BP64, 69192 Saint Fons, France

e-mail: abenaarb@univ-montp2.fr

A. Chrysochoos

LMGC, CNRS, Université Montpellier 2, Place Eugène Bataillon, 34095 Montpellier, France

G. Robert

Solvay Engineering Plastics, Technyl Innovation Center-Simulation et Validation des Applications, Avenue Ramboz - BP64, 69192 Saint Fons, France 
Many experimental surveys based on energy concepts are needed to improve constitutive material models, thus facilitating the use and application of such engineering materials. More precisely, estimations on stored and dissipated energies may provide crucial information on microstructural changes and the nature of their irreversibility. The most significant developments in the computation and interpretation of stored and dissipated energies are closely related to calorimetric procedures. The earliest published studies on this subject were focused on metallic materials. Farren and Taylor (1925) [1] and Taylor and Quinney (1934) [2] were the first to build an apparatus to measure dissipated energy during the deformation of metallic materials subjected to quasi-static monotonous tensile tests. Williams (1967) [3] and Leach (1970) [4] reviewed calorimetric methods in detail and assessed calorimetry procedures and equipment applicable for heat energy measurement. Most investigations have focused on the Taylor-Quinney ratio, which expresses the fraction of the anelastic deformation energy rate irreversibly converted into heat, i.e. dissipated. In addition, these early works were limited by the fact that they were mostly carried out with standard and traditional measurement devices that could not reliably obtain high precision estimates of this ratio. However, with the introduction of high resolution infrared scanners in the early 1980s, extensive experimental studies rectified these deficiencies and assessed the Taylor-Quinney coefficient with greater accuracy (see e.g. [5-10]).

In the present paper, we document some findings pertaining to the thermomechanical behavior of a PA6.6 matrix having different hygrothermal states (relative humidity: dry-as-molded, RH50 and RH80). Specifically, we used infrared and CCD cameras to simultaneously record, during cyclic loading, fields corresponding to temperature variations and displacement over the sample gauge part. We focused particularly on the energy balance form associated with the mechanical hysteresis loop. In particular, we obtained energy balances at two different loading frequencies, thus providing the deformation, dissipated and stored energy time patterns. We finally derived time courses of the Taylor-Quinney ratio in order to quantify the relative importance of both mean dissipated and stored energy rates over each complete loading cycle.

In the following, we briefly outline the theoretical framework used to interpret the experiments. We then describe the experimental setup and the tests performed. Finally, the main energy results are shown and discussed with respect to the mechanical hysteretic response.

\subsection{Governing Equations}

\subsubsection{Energy Balance}

Energy storage depicts the internal energy changes induced by anelastic transformations. An essential feature of this stored energy is that it remains in the deformed material, at least temporarily, until possible release during restoration processes. Generally, the stored energy amounts are obtained as the difference between the deformation energy spent during the material transformation and the dissipated energy. The total deformation energy $\mathcal{W}_{\text {def }}$ is usually determined from forcedisplacement data (and/or from stress-strain data for local analyses). This deformation energy can be split into the elastic $\mathcal{W}_{\mathrm{e}}$ and anelastic $\mathcal{W}_{\text {an }}$ energies, where $\mathcal{W}_{\text {an }}$ is both stored and dissipated. In this context, the stored energy rate $\mathcal{W}_{\mathrm{s}}^{\prime}$ reads [5]:

$$
\mathcal{W}_{\mathrm{s}}^{\prime}=\mathcal{W}_{\mathrm{def}}^{\prime}-\mathcal{W}_{\mathrm{e}}^{\prime}-\mathcal{D}_{1}
$$

According to the energy rate balance equation Eq. 23.1, a correct estimate of $\mathcal{W}_{\text {def }}^{\prime}, \mathcal{D}_{1}, \mathcal{W}_{\mathrm{e}}^{\prime}$ leads to indirect assessment of $\mathcal{W}_{\mathrm{s}}^{\prime}$. Since the mechanical and heat energies are measured by two different techniques, systematic errors in one of the quantities do not cancel systematic errors in the measurement of stored energy. In the following, only the assessment over each complete cycle of the above volume energy rates will be considered. Thus, the elastic component per cycle remains close to zero and it can be considered that the mechanical and anelastic energy rates per unit volume per cycle are approximately the same $\left(\mathcal{W}_{\text {def }}^{\prime}\right)^{(n)} \approx\left(\mathcal{W}_{\text {an }}^{\prime}\right)^{(n)}$ (n representing the considered cycle). The key issue here not only concerns computing the stored energy rate per unit volume and per cycle but also evaluating its contribution in the global energy balance. We thus define the variable stored energy rate ratio which is expressed by $\beta_{\mathrm{s}}^{(\mathrm{n})}=\left(\mathcal{W}_{\mathrm{s}}^{\prime}\right)^{(\mathrm{n})} /\left(\mathcal{W}_{\text {def }}^{\prime}\right)^{(\mathrm{n})}$. The time courses of this ratio will be shown and analyzed in the following. Note that $\beta_{\mathrm{s}}^{(\mathrm{n})}$ can be related to $\beta_{\text {diff }}^{(\mathrm{n})}$, introduced by Rittel in [8], to describe the dissipated part of the deformation energy rate: $\beta_{\text {diff }}^{(\mathrm{n})}=1-\beta_{\mathrm{s}}^{(\mathrm{n})}$. 


\subsubsection{Thermodynamic Foundations of the Heat Equation}

The Thermodynamics of Irreversible Processes framework [11] is hereafter used to introduce the energy terms. Under the hypothesis of small deformations, the thermal, mechanical and microstructural states of the material are described by the following observable state variables: the absolute temperature $T=\alpha_{\mathrm{o}}$, the small strain tensor $\boldsymbol{\varepsilon}=\boldsymbol{\alpha}_{1}$ and a set of internal variables $\left\{\alpha_{\mathrm{k}}\right\}_{\mathrm{k}=1, \ldots, \mathrm{m}}$. The chosen thermodynamic potential is the specific Helmholtz free energy $\psi=\psi\left(T, \boldsymbol{\varepsilon}, \alpha_{\mathrm{k}}\right)$. Using the scalar product of the following "flux" vector $\left(\dot{\boldsymbol{\varepsilon}}, \dot{\alpha}_{\mathrm{k}}, \mathbf{q}\right)$ and its conjugate vector of thermodynamic forces $\left(\boldsymbol{\sigma}-\rho \boldsymbol{\psi}_{, \varepsilon}, \rho \psi_{, \alpha_{\mathrm{k}}},-T^{-1} \operatorname{grad} T\right)$, the classical Clausius-Duhem inequality leads to [11]:

$$
\mathcal{D}=\underbrace{\left(\boldsymbol{\sigma}-\rho \boldsymbol{\psi}_{, \boldsymbol{\varepsilon}}\right): \dot{\boldsymbol{\varepsilon}}-\rho \psi_{, \alpha_{\mathrm{k}}} \dot{\alpha}_{\mathrm{k}}}_{\mathcal{D}_{1}} \underbrace{-T^{-1} \operatorname{grad} T . \mathbf{q}}_{\mathcal{D}_{2}} \geq 0
$$

where $\boldsymbol{\sigma}, \mathbf{q}$ and $\rho$ are the total stress tensor, the heat flux vector and the material density, respectively, while $\dot{\mathbf{x}}$ stands for the time derivative of the state variable $\mathbf{x}$, and the operator : denotes the tensorial contraction over two indices.

We assume generally that the intrinsic $\mathcal{D}_{1}$ and thermal $\mathcal{D}_{2}$ dissipation are separately non-negative, i.e. $\mathcal{D}_{1} \geq 0$ and $\mathcal{D}_{2} \geq 0$. Next, we define the specific heat as $C_{\boldsymbol{\varepsilon}, \alpha}=-T \psi_{, T T}$ at constant $\boldsymbol{\varepsilon}$ and $\alpha_{\mathrm{k}}$. By combining both principles of thermodynamics, the standard local heat diffusion equation can be directly obtained as follows:

$$
\rho C_{\varepsilon, \boldsymbol{\alpha}} \dot{T}+\operatorname{div}(\underbrace{-\boldsymbol{k} \mathbf{g r a d} T}_{\mathbf{q}})=\underbrace{\left(\boldsymbol{\sigma}-\rho \boldsymbol{\psi},_{\varepsilon}\right): \dot{\boldsymbol{\varepsilon}}-\rho \psi, \alpha_{k} \dot{\alpha}_{k}}_{\mathcal{D}_{1}}+\underbrace{\rho T \psi \boldsymbol{\psi}_{T, \varepsilon}: \dot{\boldsymbol{\varepsilon}}+\rho T \psi, T, \alpha_{k} \dot{\alpha}_{k}}_{w_{c}^{\prime}}+r_{e}
$$

where $\boldsymbol{k}$ denotes the heat conduction tensor and $r_{\mathrm{e}}$ the heat supply term characterizing the volume heat exchange with the surroundings.

The terms on the left hand side represent a partial differential operator applied to the temperature. The thermal inertia $\rho C_{\varepsilon, \alpha} \dot{T}$ reflects the stored or released heat rate. The term $-\operatorname{div}(\boldsymbol{k} \operatorname{grad} T)$ stands for the volume heat loss by conduction. The terms on the right hand side pool the different heat sources. In turn, we find the intrinsic mechanical dissipation $\mathcal{D}_{1}$, which reflects the irreversible transformation into heat of the deformation energy, the thermomechanical coupling sources $\mathcal{W}_{c}^{n}$, which are the calorific signatures of possible interactions between temperature and the other state mechanical or microstructural variables.

Under some reasonable hypotheses, the previous heat balance equation Eq. 23.3 can be reduced to three different simplified formulations, i.e. two, one and/or zero-dimensional diffusion equations. Readers interested in these formulations are referred to $[12,13]$. The choice of a particular model strongly depends on the type of experimental investigation under consideration. The passage from a $2 \mathrm{D}$ to a $0 \mathrm{D}$ model is possible since heat source fields remain homogeneous throughout the test. By contrast with the 2D and/or 1D diffusion models, the 0D heat equation is less sensitive to measurement noise and less time consuming in terms of computation duration. This approach is well adapted to so-called homogeneous tests when a rapid experimental estimate of heat sources is required. This global approach is generally based on the hypothesis that for uniaxial tests the heat source distributions are uniform throughout the test duration within the specimen gauge area. In the following, $\theta$ denotes the spatial average of temperature variations over the gauge part ( $\mathrm{x}, \mathrm{y}$ and $\mathrm{z}$ coordinates). The local heat diffusion equation Eq. 23.3 can be simplified to the following differential equation:

$$
\rho C_{\varepsilon, \alpha}\left(\dot{\theta}+\frac{\theta}{\tau_{\text {th }}^{\text {od }}}\right)=\mathcal{D}_{1}+\mathcal{W}_{\text {ther }}^{\prime}
$$

where $\tau_{\mathrm{th}}^{\text {Od }}$ represents a time constant characterizing the linear heat losses perpendicular to the specimen surface, while the $\mathcal{W}_{\text {ther }}^{\prime}$ term denotes the thermoelastic coupling source. 


\subsection{Experimental Procedure}

The experimental setup used for conducting the thermomechanical tests is shown in Fig. 23.1. Samples were loaded using an MTS 810 hydraulic testing machine equipped with a load cell of $\pm 25 \mathrm{kN}$. Temperature variations on one side and displacements on the other side of the sample were measured and captured simultaneously using both a high resolution infrared focal plane array camera and a high resolution visible CCD camera, respectively.

\subsubsection{IRT Techniques}

Infrared thermography is a convenient non-contact imaging technique that uses an infrared matrix element to visualize and estimate temperature fields of material surfaces. The principle of the technique is based on use of the intensity and wavelength of radiation emitted by the loaded material to measure its surface temperature according to Planck's law. In this survey, the infrared thermal imaging system used for thermographic measurement was a CEDIP Jade camera, cooled with a Stirling cooler. This contained InSb matrix elements which are sensitive to infrared radiation in the 3-5 $\mu \mathrm{m}$ wavelength range. The camera was equipped with a $25 \mathrm{~mm}$ lens and positioned on a tripod approximately $0.65 \mathrm{~m}$ from the specimen surface. We decided to operate the detector with a $160 \times 128$ pixel window size, $29 \mu \mathrm{m}$ pixel size and $400 \mu \mathrm{s}$ integration time. The IR camera frame rate was set at tenfold the loading frequency to obtain enough sampled data per cycle and to improve the signal-to-noise ratio using local least-squares fitting.

During the experiments, several factors complicated measurement of the surface temperature of the investigated materials. Several experimental precautions were thus taken to overcome these difficulties. For instance, the heat flux density transferred to the infrared sensor included noise components due to the reflected incident energy from the surroundings. The IR measurements were therefore carried out indoors, and the experimental tools were screened and isolated far from the radiation sources of the surroundings. Besides, the camera was turned on for $4 \mathrm{~h}$ before starting the experiments in order to reach thermal equilibrium. The temperature was quantitatively measured after improving the surface emissivity. The tested specimens were coated with matt black paint, and we assumed that the specimens were as perfect as possible black bodies with an emissivity value close to 1 . Even though these precautions were taken, the measured data were not necessarily quantitatively reliable and the true temperature value could not be derived directly. An appropriate calibration protocol was thus used to determine the true temperature value. This allowed conversion of the thermal radiation digitized by the infrared camera into temperature using pixel calibration functions [14]. This pixel protocol was based on a polynomial fitting of the digital levels $\mathcal{D} \mathcal{L}_{(m, n)}$ delivered by each individual detector element $(m, n)$ using a black body

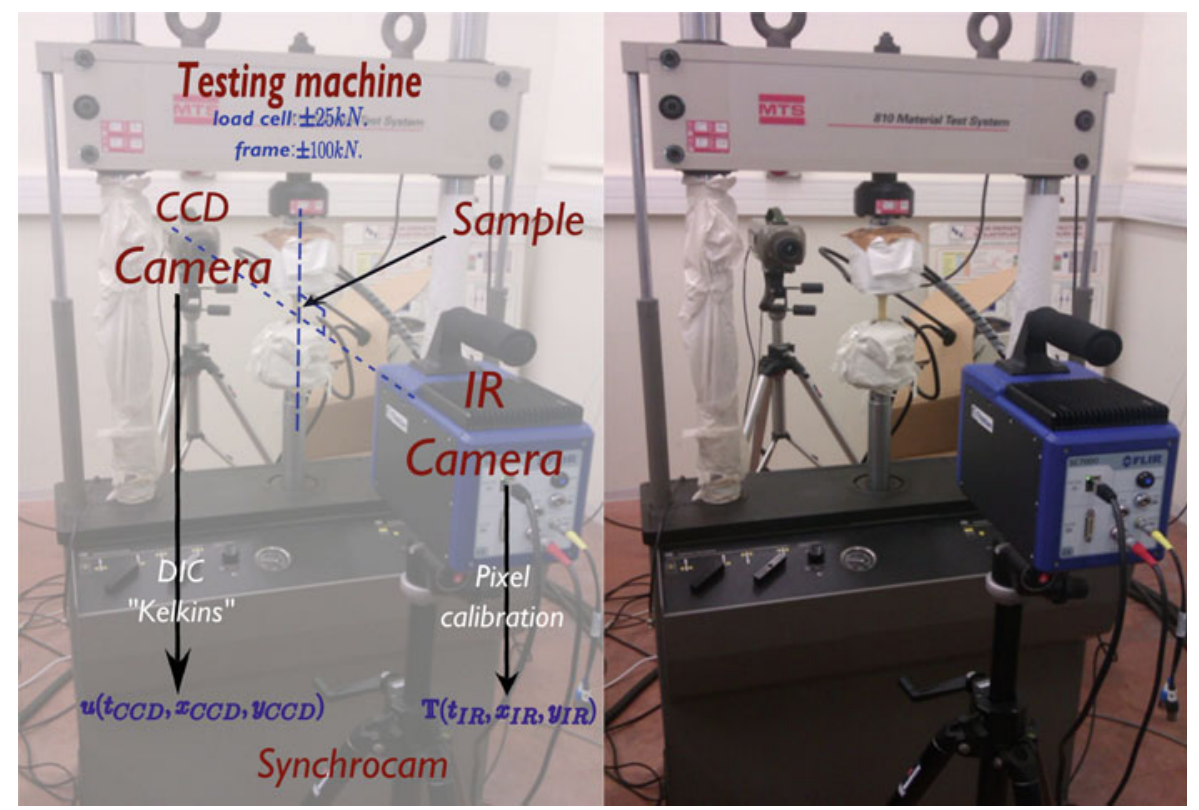

Fig. 23.1 Experimental setup showing both cameras used for recording the in-plane displacement and temperature fields 
with a uniform high emissivity coating, maintained at cold and hot temperatures $T_{(m, n)}$. The measurement accuracy could thus be assessed for each pixel individually in the resulting image.

$$
T_{(m, n)}=\sum_{k=0}^{d_{f}} x_{(m, n) \mathbf{k}} \mathcal{D} \mathcal{L}_{(m, n)}
$$

where $d_{f}$ denotes the degree of the polynomial fitting function and $x_{(m, n)}$ are coefficients of the calibration law for the element $(m, n)$. These coefficients were derived from least squares fitting approximations.

Two bad pixel detection criteria were used. The first consisted of the elimination of pixels whose response predicted by the polynomial fitting $T_{(m, n)}$ was far from the ordered temperature of the used black body $T_{b b}$. Whereas the thermal response of the second eliminated pixels was too far from the calculated average temperature throughout the detector $T_{a v}$. In agreement with the foregoing, both criteria were written as:

$$
\left|T_{(m, n)-} T_{b b}\right| \geq \delta T_{1},\left|T_{(m, n)-} T_{a v}\right| \geq \delta T_{2}
$$

where $\delta T_{1}$ and $\delta T_{2}$ were respectively the threshold values of the first and second criteria.

The room temperature was taken into account by recording the temperature data of an unloaded specimen (dummy) kept nearby the test specimen. The temperature images were then acquired using Altair software and stored on a computer hard drive. A local least-square fitting method using an optimized approximation function set was developed to filter the IR images from the noise [15].

\subsubsection{DIC Techniques}

Digital image correlation techniques (DIC) are now well-known non-contact full-field measurement tools which offer the possibility of determining displacement and strain fields at the surface of loaded specimens. It consists of capturing consecutive images of a random surface pattern recorded using a charge coupled device (CCD) camera. To apply this method, the random pattern (local optical signature of a material surface element) was achieved by spraying the specimen surface with a painted speckle pattern (pulverization of black and white paints). These patterns were chosen in order to obtain well illuminated visible images with high contrast. The change in surface characteristics of the specimen was evaluated by matching different sub-cells of two images captured before and after loading the specimen. As it is almost impossible to find the matched zone using few pixels, a subset with multiple pixel points was defined to perform the matching process. Adequate DIC software was required to obtain information on the positional changes in the material surface element under investigation. In our case, we used the tailored Kelkins software package developed by LMGC. Displacement fields were thus determined with suitable algorithms after digitizing images captured by the visible camera [16]. Since the experimental data were inherently noisy, appropriate smoothing methods were needed to reduce the effect of noise on the strain estimate. As for the thermal data, we used projections of data on a local analytical function set in a leastsquares sense: (1) to approximate the experimental displacement values in the selected regions, (2) to reduce the noise present in these displacements, and (3) to derive the measured strain and strain rate.

\subsubsection{Synchronization System}

To monitor the energy behavior of studied materials, it is crucial to assess the applied load, temperature and strain at the same time. Therefore, it is of paramount importance to synchronize the IR and visible cameras with the load cell sensor of the hydraulic testing machine (MTS). A specific tailored electronic device named synchrocam was designed to simultaneously capture the three data types. Both imaging systems were then triggered from a digital pulse to capture data concurrently. This ensured that all data captured by the imaging systems and MTS were initiated at the same time. Fortunately, in the current investigation both systems were initiated at the same time with identical frame rates, which facilitated the image processing. 
In addition, the geometric transformation between the frames of reference of the two cameras was determined using a calibrated target. The correspondence between the spatial coordinates of the thermal image $\left(x_{I R}, y_{I R}\right)$ and those of the visible image $\left(x_{C C D}, y_{C C D}\right)$ was established by trigging the motion of the calibrated target using the following equations:

$$
\left(\begin{array}{l}
x_{C C D} \\
y_{C C D}
\end{array}\right)=\underbrace{\left(\begin{array}{ll}
\gamma_{x} & \eta_{x} \\
\gamma_{y} & \eta_{y}
\end{array}\right)}_{\mathcal{R}}\left(\begin{array}{l}
x_{I R} \\
y_{I R}
\end{array}\right)+\underbrace{\left(\begin{array}{l}
\lambda_{x} \\
\lambda_{y}
\end{array}\right)}_{\mathcal{D}}
$$

where $\mathcal{R}$ is the rotation matrix and $\mathcal{D}$ a displacement vector. This spatial correspondence was carried out before each new test.

\subsection{Experimental Results}

The experimental study involved observation and analysis of the thermomechanical and energy properties of the PA6.6 matrix involved during cyclic fatigue tests. The investigations were essentially divided into two parts. At two different loading frequencies, the first part examined the contribution of the stored and dissipated energies in the global energy balance associated with dry specimens of polyamide 6.6. The second part was focused on highlighting the effect of relative humidity on PA6.6 specimens having different hygrothermal states (relative humidity: dry-as-molded, RH50 and RH80). The cyclic tensile-tensile loads were carried out under a stress ratio of 0.1 . The loading frequency ranged from $1 \mathrm{to} 10 \mathrm{~Hz}$, while the number of cycles was $10^{4}$. The cycle number was high enough to raise the kinematic and thermal properties of the investigated materials.

\subsubsection{Thermal Response}

For the sake of simplicity, the mean temperature variations averaged over the sample gauge part are first considered. Typical plots associated with these thermal variations as a function of time (image number) and at loading rates of 1 and $10 \mathrm{~Hz}$ and for the same stress range $\Delta \sigma$ are shown in Fig. 23.2. For confidentiality reasons, the stress range will not be given.

Conventionally, temperature variations involve low drifts with an oscillation in opposite phase with the monochromatic loading signal. We associated two independent heat sources with both of these thermosignal characteristics. The oscillation is induced by the thermoelastic source coming from the material thermodilatability. The progressive heating of the specimen is associated with the dissipated energy. It is often characterized by a gradual global increase in temperature variations (blue dashed line in Fig. 23.2). A decrease in mean temperature is associated with a decrease in the dissipation intensities, with the heat exchange coefficients between the specimen and the surroundings assumed to be constant throughout the test. Comparing the trend of thermal curves in dry-as-moulded speciments subjected to low (Fig. 23.2, plot (a)) and high loading rates (Fig. 23.2, plot (b)), it was shown that the temperature variation values were similar throughout the test. For the same

Fig. 23.2 Spatial averages of temperature variations showing both dissipation and coupling sources for dry PA6.6 matrix specimens subjected to (a) low loading frequency of $1 \mathrm{~Hz}$, (b) high loading frequency of $10 \mathrm{~Hz}$, while the stress ratio was $R_{\sigma}=0.1$
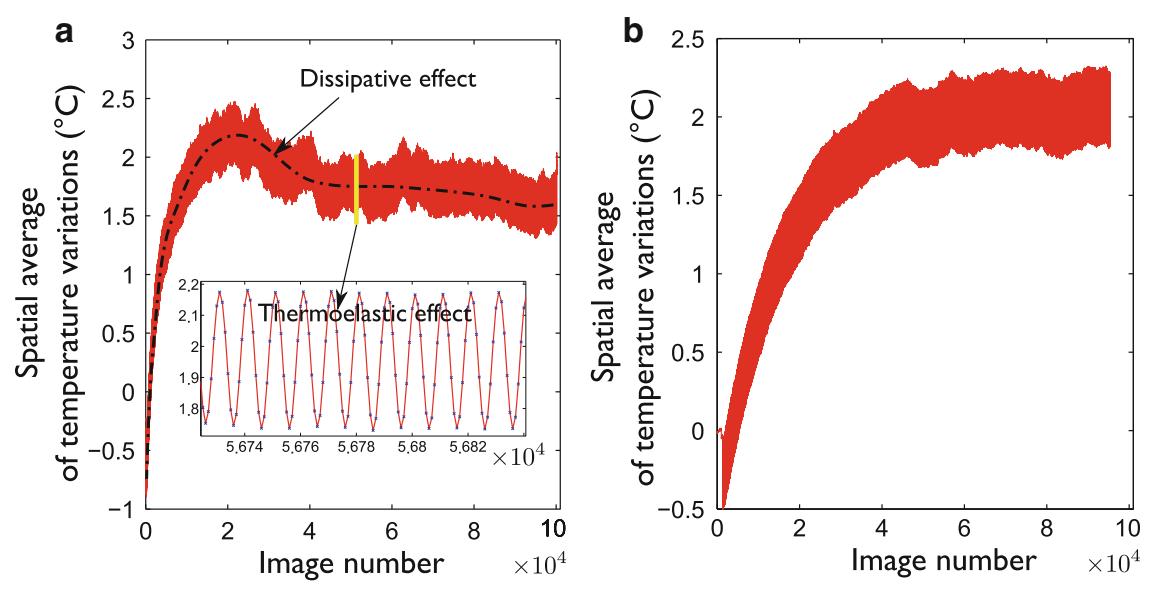
stress range, the thermoelastic amplitude was the same for both low and high loading frequencies with induced temperature variations of $0.4{ }^{\circ} \mathrm{C}$. However, the self heating induced by the dissipative effect was about $2{ }^{\circ} \mathrm{C}$. From a calorimetric viewpoint, it was found that the intrinsic dissipation intensities remained very low compared to the thermoelastic source ranges.

\subsubsection{Energy Rate Balance}

\subsubsection{Loading Frequency Effect}

The energy balances were established for 1 and $10 \mathrm{~Hz}$ loading frequencies. All experimental data were converted and used to compute the previous energies used in Eq. 23.4. The dark curve corresponds to the mean deformation energy rate per cycle, the green curve shows values of the stored energy rate per cycle and the red curve indicates the values of the mean intrinsic dissipation per cycle. All energies are plotted as a function of the cycling number, and the time courses associated with their variable ratios are given in Figs. 23.3, plots (b, d) and 23.4. Note that the heat sources were divided by $\rho C$ and thus expressed in ${ }^{\circ} \mathrm{C} / \mathrm{s}$ in order to define, for each energy, an equivalent heating rate, thus facilitating comparisons between the dissipative and stored energy rates.

From all the plots, it can be observed that the overall shape of the energy rate balance closely depends on the loading frequency. For a dry matrix at a high loading rate $\left(f_{L}=10 \mathrm{~Hz}\right)$, the results indicate a substantial part of the mean stored energy rate per cycle with a ratio near $83 \%$ (Fig. 23.3, plot (b)). This part dominates the mean intrinsic dissipation per cycle whose
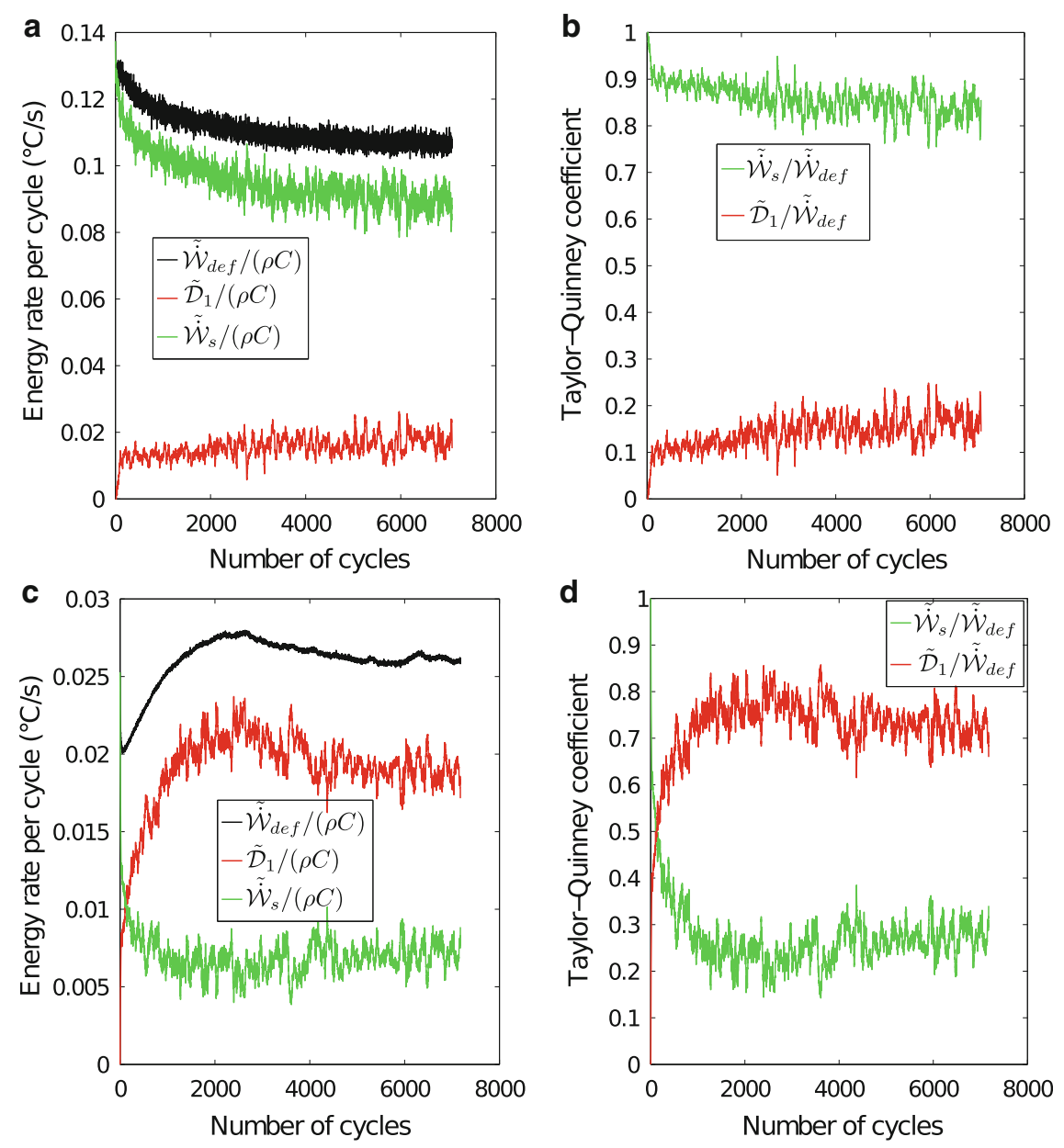

Fig. 23.3 The energy rate balance for a PA6.6 dry matrix subjected to tensile-tensile tests: (a) loading rate $f_{L}=10 \mathrm{~Hz}$, (b) corresponding TaylorQuinney, (c) loading rate $f_{L}=1 \mathrm{~Hz}$, (b) corresponding Taylor-Quinney. The stress ratio is $R_{\sigma}=0.1$ 
Fig. 23.4 The energy rate balance for a PA6.6 dry matrix from the beginning of cyclic loading until the last few cycles before the macroscopic crack. The stress ratio is $R_{\sigma}=0.1$
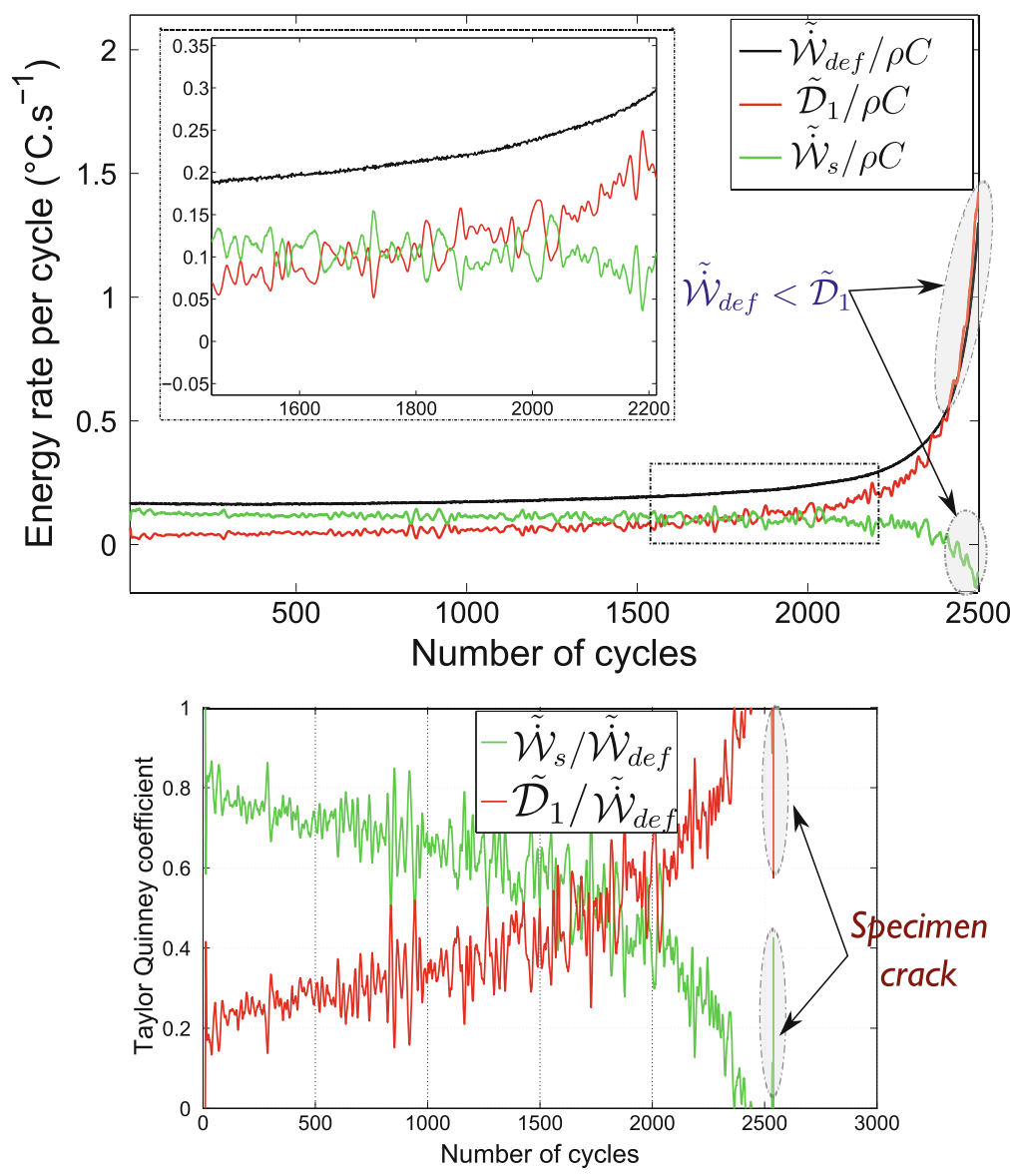

ratio seems to level off at around $17 \%$. However, in the case of low loading frequencies $\left(f_{L}=1 \mathrm{~Hz}\right)$, the trend of these variable ratios changes completely (Fig. 23.3). The mean intrinsic dissipation per cycle is now predominant compared to the stored energy rate. It starts increasing at the beginning of loading and then stabilizes after the first thousands of cycles, with a ratio of $73 \%$ (Fig. 23.3, plot (b)) and $65 \%$ (Fig. 23.3, plot (d)). Note that this energy stabilization was never accompanied by a stabilized cyclic shakedown, a ratcheting behavior induced by the mean stress effect, leading to a regular drift in the cyclic hysteresis loop.

Our studies so far have shown that the mean mechanical energy over a complete cycle exceeded the heat generated. The strained sample could generally not dissipate more heat than it received. However, in some cases the heat was found to be generated at a higher rate than the mechanical energy, indicating a release of stored energy (see e.g. [8, 17, 18] for polymers and [19] for metals). Fig. 23.4 illustrates a case of a dry PA6.6 specimen that released more heat than the mechanical energy at the last stages of its fatigue life. Thermal and kinetic images were recorded until final rupture of the specimen (2,531 cycles), allowing us to study the trend of the global energy balance close to the macroscopic crack. In this figure, the ratio of the stored energy rate over a complete cycle falls continuously with the test time. The stored energy rate is high at the beginning of loading and then decreases as the test proceeds until reaching negative values at the end of loading. This highlights a rapid increase in intrinsic dissipation, thus indicating an amount of internal energy converted into heat. Even at low frequencies, the stored proportion remains high and could thus represent a slow but regular material degradation.

\subsubsection{Relative Humidity Effect}

In what follows, we set the loading rate at $10 \mathrm{~Hz}$ and we highlighted the effect of relative humidity upon the kinetics and contributions of stored and dissipated energy rates. For a dry-as-molded matrix, the results indicated a substantial part of the mean stored energy rate per cycle with a stored ratio near $70 \%$ (see Fig. 23.5, plot (a)). This part dominated the mean intrinsic dissipation per cycle whose ratio seemed to be stabilized at around $30 \%$. This finding is compatible with that 
a

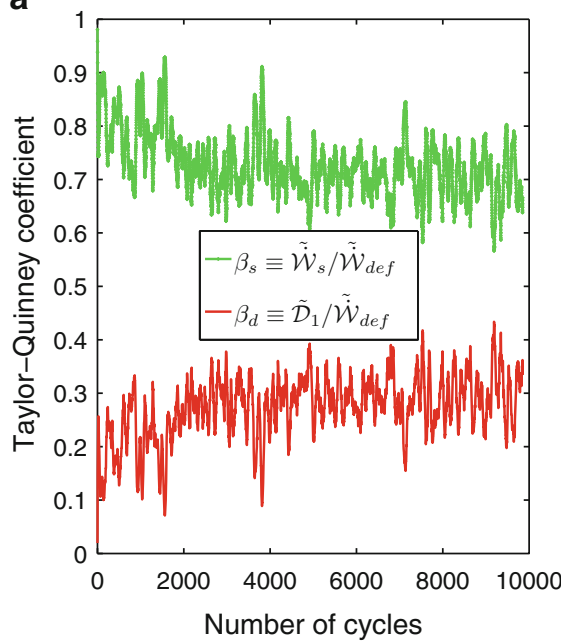

b

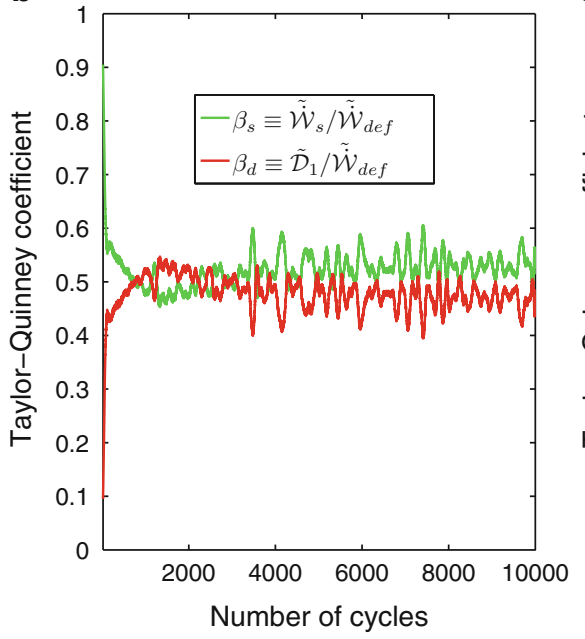

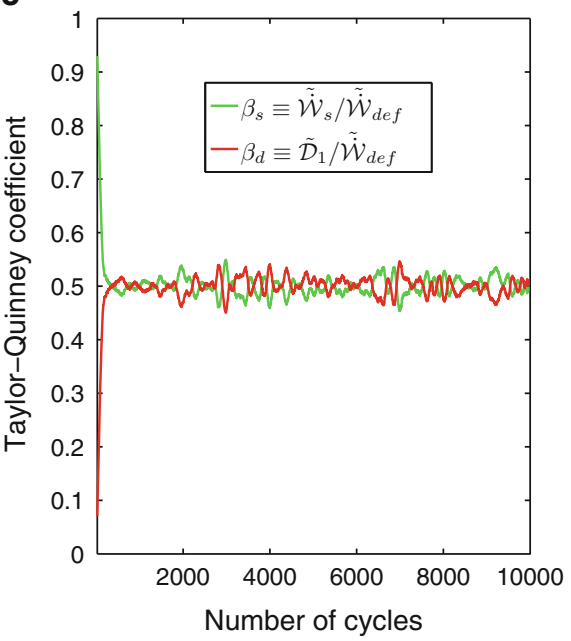

Fig. 23.5 Taylor-Quinney coefficients for the PA6.6 matrix subjected to a loading frequency of $10 \mathrm{~Hz}$. The plots show results coming from (a) dry-as molded PA6.6 matrix, (b) specimen preconditioned at RH50 and (c) specimen preconditioned at RH80. The stress ratio $R_{\sigma}=0.1$

obtained in Fig. 23.3, plot (a). Whereas, the results indicated in Fig. 23.5, plots (b, c) showed that ratios decreased at the beginning of loading and stabilized continuously after a thousand of cycles. The comparison in terms of Taylor-Quinney ratios for dry-as-molded and conditioned specimens indicated that for a certain relative humidity level, the stored energy contribution was not modified by the changing water content, as demonstrated by experiments on both RH50 and RH80. For RH50 specimens, the mean intrinsic dissipation ratio increased at the beginning of loading and then stabilized after the first few cycles, with a value of almost $50 \%$ (see Fig. 23.5, plot (b)). This ratio had approximately the same value for specimens conditioned at RH80 (see Fig. 23.5, plot (c)). The deformation energy rate appeared to be equally converted to both dissipated and stored energy rates.

\subsection{Concluding Comments}

Some aspects related to thermal and energy responses associated with PA6.6 matrix specimens subjected to tensile-tensile tests were investigated here. The first aspect was the influence of the loading frequency on the energy balance form. The investigations of energy stored during deformation indicated that the stored ratio was significantly lower at low loading rates (e.g. $25 \%$ ) than those obtained at high loading rates (e.g. $80 \%$ ). In addition, it was shown that this ratio could be negative at the last fatigue stages, heralding the macroscopic crack. This negative value was interpreted as a transformation of stored into dissipated energy.

Taylor-Quinney ratios were also determined for three different hygrothermal states (RH0, RH50 and RH80). This generated some insight into the water plasticizing effect through values of the stored energy rate. For dry-as-molded and conditioned specimens, the Taylor-Quinney ratios indicated a limited influence of the relative humidity which seemed to saturate for RH greater than RH50. In other words, the stored energy amounts were no longer modified by the water content change, as shown in both experiments of conditioned specimens (RH50 and RH80), for which we obtained the same stored energy ratio. Further experiments are required to obtain more information on the humidity effects, especially in fiberreinforced polymeric materials (DURAFIP project) in which interactions between the fiber orientation and thermo-hygromechanical matrix behavior have to be explored. Experiments are currently under way to gain further insight into the role of loading frequency and humidity on the cyclic behavior of these industrial composites.

Acknowledgements The authors gratefully acknowledge Solvay Engineering Plastics for supporting this work and for providing material data and specimens. This work benefited from the financial support of the French Minister for Research (ANRT) and was performed in the framework of the European DURAFIP project 


\section{References}

1. Farren WS, Taylor GI (1925) The heat developed during plastic extension of metals. Proc R Soc A 107:422-451

2. Taylor GI, Quinney H (1934) The latent energy remaining in a metal after cold working. Proc R Soc A 14(3):307-326

3. Williams RO (1967) In: Herman H (ed) Experimental methods of materials research, vol 1. Interscience, New York, NY

4. J. S. LI. Leach (1970) Physicochemical measurements in metals research, vol 4. R.A. Rapp (ed) Interscience, New York, NY

5. Chrysochoos A (1985) Energy balance for elastic plastic deformation at finite strain (in French). J Theor Appl Mech 5:589-614

6. Chrysochoos A, Maisonneuve O, Martin G, Caumon H, Chezeaux JC (1989) Plastic and dissipated work and stored energy. Nucl Eng Des 114:323-333

7. Mason J, Rosakis A, Ravichandran G (1994) On the strain and strain rate dependence of the fraction of plastic work converted to heat: : an experimental study using high speed infrared detectors and the Kolsky bar. Mech Mater 17:135-145

8. Rittel D (1999) On the conversion of plastic work to heat during high strain rate deformation of glassy polymers. Mech Mater 31:131-139

9. Rosakis P, Rosakis AJ, Ravichandran G, Hodowany J (2000) A thermodynamic internal variable model for the partition of plastic work into heat and stored energy in metals. J Mech Phys Solids 48:581-607

10. Oliferuk W, Maj M, Raniecki B (2004) Experimental analysis of energy storage rate components during tensile deformation of polycrystals. Mater Sci Eng A 374:77-81

11. Halphen B, Nguyen QS (1975) On the generalized standards materials (in French). Journal de Mécanique 14(I):39-63

12. Chrysochoos A, Louche H (2000) An infrared image processing to analyse the calorific effects accompanying strain localisation. Int J Eng Sci 38:1759-1788

13. Berthel B, Chrysochoos A, Wattrisse B, Galtier A (2008) Infrared image processing for the calorimetric analysis of fatigue phenomena. Exp Mech 48:79-90

14. Honorat V, Moreau S, Muracciole JM, Wattrisse B, Chrysochoos A (2005) Calorimetric analysis of polymer behaviour using a pixel calibration of an IRFPA camera. Qirt J 2:153-171

15. Boulanger T, Chrysochoos A, Mabru C, Galtier A (2004) Calorimetric analysis of dissipative and thermoelastic effects associated with the fatigue behavior of steels. Int J Fatigue 26:221-229

16. Wattrisse B, Chrysochoos A, Muracciole J-M, Némoz-Gaillard M (2001) Analysis of strain localization during tensile tests by digital image correlation. Exp Mech 41:29-39

17. Dillon OWJ (1966) The heat generated during the torsional oscillations of copper tubes. Int J Solids Struct 2:181-204

18. Benaarbia A, Chrysochoos A, Robert G (2014) Kinetics of stored and dissipated energies associated with cyclic loadings of dry polyamide 6.6 specimens. Polym Test 34:155-167

19. Oliferuk W, Korbel A, Bochniak W (2001) Energy balance and macroscopic strain localization during plastic deformation of polycrystalline metals. Mater Sci Eng A 319:250-253 